\title{
BMJ Open COVID-19 hinterland: surveilling the self-reported impacts of the pandemic on diabetes management in the USA (cross-sectional results of the iNPHORM study)
}

\author{
Alexandria A Ratzki-Leewing, ${ }^{1}$ Bridget L Ryan, ${ }^{1,2}$ John D Buchenberger, ${ }^{3}$ \\ Joseph W Dickens, ${ }^{4}$ Jason E Black, ${ }^{2}$ Stewart B Harris (I) ${ }^{1,2}$
}

To cite: Ratzki-Leewing AA, Ryan BL, Buchenberger JD, et al. COVID-19 hinterland: surveilling the self-reported impacts of the pandemic on diabetes management in the USA (cross-sectional results of the iNPHORM study). BMJ Open 2021;11:e049782. doi:10.1136/ bmjopen-2021-049782

- Prepublication history and additional supplemental material for this paper are available online. To view these files, please visit the journal online (http://dx.doi.org/10.1136/ bmjopen-2021-049782)

Received 04 February 2021 Accepted 17 August 2021

Check for updates

(c) Author(s) (or their employer(s)) 2021. Re-use permitted under CC BY-NC. No commercial re-use. See rights and permissions. Published by BMJ.

${ }^{1}$ Department of Epidemiology and Biostatistics, Schulich

School of Medicine and Dentistry, Western University, London, Ontario, Canada ${ }^{2}$ Department of Family Medicine, Western University Schulich School of Medicine and Dentistry, London, Ontario, Canada

${ }^{3}$ Ipsos Healthcare, Ipsos, New York, New York, USA ${ }^{4} \mathrm{SMX}$, Ipsos, Chicago, Illinois, USA

Correspondence to Dr Stewart B Harris; sharris1@uwo.ca

\section{ABSTRACT}

Main objective To determine how and to what extent COVID-19 has affected real-world, self-reported glycaemic management in Americans with type 1 or type 2 diabetes taking insulin and/or secretagogues, with or without infection.

Design A cross-sectional substudy using data from the Investigating Novel Predictions of Hypoglycemia Occurrence using Real-world Models panel survey. Setting USA.

Participants Americans 18-90 years old with type 1 or 2 diabetes taking insulin and/or secretagogues were conveniently sampled from a probability-based internet panel.

Primary outcome measure A structured, COVID-19specific questionnaire was administered to assess the impact of the pandemic (irrespective of infection) on socioeconomic, behavioural/clinical and psychosocial aspects of glycaemic management.

Results Data from 667 respondents (type 1 diabetes: 18\%; type 2 diabetes: $82 \%$ ) were analysed. Almost 25\% reported $A 1 \mathrm{c}$ values $\geq 8.1 \%$. Rates of severe and nonsevere hypoglycaemia were $0.68(95 \% \mathrm{Cl} 0.5$ to 0.96$)$ and 2.75 (95\% $\mathrm{Cl} 2.4$ to 3.1 ) events per person-month, respectively. Ten respondents reported a confirmed or probable COVID-19 diagnosis. Because of the pandemic, $24 \%$ of respondents experienced difficulties affording housing; $28 \%$ struggled to maintain sufficient food to avoid hypoglycaemia; and $19 \%$ and $17 \%$ reported challenges accessing diabetes therapies and testing strips, respectively. Over one-quarter reported issues retrieving antihyperglycaemics from the pharmacy and over one-third reported challenges consulting with diabetes providers. The pandemic contributed to therapeutic non-adherence (14\%), drug rationing (17\%) and reduced monitoring (16\%). Many struggled to keep track, and in control, of hypoglycaemia (12\%-15\%) and lacked social support to help manage their risk (19\%). Nearly half reported decreased physical activity. Few statistically significant differences were observed by diabetes type.

Conclusions COVID-19 was found to cause substantial self-reported deficiencies in glycaemic management.

\section{Strengths and limitations of this study}

- This is the first US-based, primary research study to quantify the real-world, self-reported impact of the COVID-19 situation on the socioeconomic, behavioural/clinical and psychosocial aspects of glycaemic management.

- A novel COVID-19-specific questionnaire was developed and administered to a real-world cohort of Americans with type 1 and type 2 diabetes taking insulin and/or secretagogues; study participants were recruited from a large, probability-based internet panel.

- Estimates presented in this study may be conservative as they describe the early phase of the pandemic

Study results signal the need for decisive action to restabilise routine diabetes care in the USA. Trial registration number NCT04219514.

\section{INTRODUCTION}

COVID-19 is among the most devastating health crises in global history. In the USA, the first reported infection occurred on 19 January 2020. ${ }^{1}$ Since then, the number of confirmed US cases has surpassed 33.5 million, including over 605000 deaths (5 July 2021). ${ }^{2}$

People with diabetes (PWD) have been identified as clinically vulnerable to COVID19. In the USA, diabetes ranks as the second most common underlying health condition among all cases and has been connected to more severe infection. ${ }^{34}$ However, less appreciated in the literature are the disruptions caused by the pandemic on routine diabetes care. These disruptions expose those with COVID-19 and all 34+ million Americans with diabetes to poor outcomes. Understanding 
how the pandemic affects diabetes services and management is crucial to informing short and long-term clinical decision-making and public health planning. Targeted measures to help protect these Americans from the direct and indirect effects of the COVID-19 pandemic should be a top priority for all healthcare and government officials.

\section{The complex hinterland of COVID-19 and diabetes}

The pathophysiological benefits of glycaemic control on diabetes outcomes have been well established. Numerous studies have linked chronic hyperglycaemia and glycaemic variability to increased risks of microvascular and macrovascular complications and mortality. In addition, dysglycaemia can potentiate immunosuppression, ${ }^{5}$ increasing viral susceptibility and risk of poor clinical outcomes. ${ }^{6}$ While the role of coexistent diabetes in the pathogenesis of COVID-19 is still being determined, ${ }^{7}$ emerging signals suggest that euglycaemia protects against infection and severity of prognoses. ${ }^{8}{ }^{9}$ These data are consistent with evidence from other viral infections where glucose control showed to augment host immune response. ${ }^{510}$

To mitigate COVID-19 risks, several national and international organisations have published diabetes pandemic guides, urging PWD to maintain scrupulous adherence to all self-management and public health recommendations. ${ }^{78}$ Notably, the Centers for Disease Control and Prevention $(\mathrm{CDC})^{11}$ has recommended maintaining at least a 30-day supply of medication and 2-week supply of food. The American Diabetes Association (ADA) ${ }^{12}$ has advised storing blood glucose emergency supplies (ie, glucagon and ketone strips). The International Diabetes Federation ${ }^{13}$ has encouraged healthy nutrition and regular monitoring to help avoid the complications of high and low blood glucose.

However, the COVID-19 situation has created a challenging terrain for effective glycaemic management. ${ }^{14}$ Amid pressures to flatten the pandemic curve, PWD and their clinicians may divert focus and resources away from diabetes management, resulting in compromised care. ${ }^{8}$ Moreover, home quarantine, physical distancing and community containment-while enacted to ensure the safety of Americans-can erode chronic disease services and make it increasingly difficult for PWD to access medical supplies and engage in optimal self-management behaviour (eg, healthy eating and physical activity). ${ }^{15}$ Previous outbreaks have also been associated with inadequate diabetes monitoring and barriers to accessing healthcare, medications and testing supplies. ${ }^{4}$ Such disruptions to routine care can lead to worse glycaemic outcomes during and after the event. ${ }^{1617}$

Yet, to date, most diabetes-related COVID-19 studies in the USA have focused exclusively on the epidemiology of hospitalised cases ${ }^{18} 19$ and failed to consider how community-based chronic diabetes management has suffered in the face of the pandemic. The lack of realworld evidence on the situational effects of COVID-19 bodes ill for the implementation of effective outbreak strategies that support Americans with diabetes. As the pandemic persists into the foreseeable future, the need to address this gap only intensifies.

The main objective of this investigation was to measure how, and the extent to which, the COVID-19 situation has affected self-reported glycaemic management in the general community population of Americans with type 1 and 2 diabetes. In so doing, we aimed to chart the complex hinterland of COVID-19 as it intersects with America's other deadly epidemic: diabetes. The results of this study will be instructive for handling chronic disease management both during the current public health emergency and in future.

\section{RESEARCH DESIGN AND METHODS Study design}

This cross-sectional study describes the results of a COVID-19-specific subquestionnaire that was administered as part of the larger Investigating Novel Predictions of Hypoglycemia Occurrence using Real-world Models (iNPHORM) panel survey: a 1-year prospective analysis of real-world hypoglycaemia risk stratification in the USA. ${ }^{20}$

\section{Participants and data collection}

iNPHORM participants were conveniently sampled from randomly selected subsets of a probability-based internet panel comprising $>10000$ Americans with type 1 diabetes and $>58000$ with type 2 diabetes ( $\geq 18$ years old). These subsets were defined based on study requirements, mainly diabetes status. Individuals in each subset were contacted via email about the study; those interested in participating were directed to complete a screening questionnaire.

Panel members 18-90 years old, living in the USA for the past year and with type 1 or 2 diabetes taking insulin and/or secretagogues were eligible to enrol. Individuals were ineligible if they were or had been pregnant within the past year, were involved in an interventional study or were unable to read/understand English. To finalise the enrolment, eligible respondents needed to provide consent and complete a baseline questionnaire. Once enrolled, participants were managed and hosted by Ipsos Interactive Services (IIS, www.ipsos.com), a leading global firm in diabetes insights and real-world survey conduct.

Respondent data were collected via the online IIS platform. In addition to the screener and baseline surveys, iNPHORM participants were asked to complete 12 prescheduled, monthly follow-up questionnaires. Follow-ups were required to be submitted within 7 days of the distribution date. Automatic reminders and notifications containing survey links were emailed throughout the prospective phase. As well, honoraria were issued in the form of e-gift cards; the incentivisation scheme (based on the quantity and timing of completed surveys) complied with social standards of reciprocity and Western University's Research Ethics Board.

Owing to the escalating severity of COVID-19 in the USA, iNPHORM follow-up questionnaires were emended after 
study commencement (at follow-up month 2) to include a COVID-19-specific subquestionnaire. The subquestionnaire assessed the community infection and the impact of the pandemic on diabetes management. Data pertaining to the first administered COVID-19 subquestionnaire (21-28 April 2020) are summarised herein.

\section{Survey instruments and variables}

iNPHORM questionnaires (screening, baseline and follow-ups (including the COVID-19 subquestionnaire)) were developed by our team of epidemiologists and clinicians in consultation with the literature. All surveys were designed to be completed in English on diverse internetequipped devices (eg, computers, smartphones, tablets). Efforts were taken to avoid double-barrelled questions, clinical jargon and value-laden or complex/ambiguous language. Additionally, each item was specified to ensure its mutual exclusivity, exhaustiveness and appropriateness of detail. When necessary, concise, clearly worded preambles, instructions and definitions (including expounding mouseover texts) were provided. Participants could take as much time as needed to reflect on items and/or review clinical documentation prior to completing the question/ survey; at any point, they could opt out of responding. Questionnaires were piloted via semistructured interviews prior to fielding.

\section{COVID-19 status}

To ascertain self-reported 1-month infection status (March to April 2020), we adapted the CDC COVID-19 case definitions (April 2020). ${ }^{21}$ Two structured items were developed to capture clinical criteria (symptoms), laboratory criteria (confirmed diagnoses) and epidemiological exposure (eg, close contact with a confirmed or suspected case; international travel). Aligning with CDC recommendations, we classified respondents as confirmed, probable or possible cases. Confirmed cases were those who reported having been formally diagnosed with COVID-19. Probable cases were those who did not have a formal diagnosis but who reported (1) symptoms typical of COVID-19 and (2) $\geq 1$ form of epidemiological exposure. If only one of the two latter conditions was met, we classified individuals as possible cases.

\section{Impact of the COVID-19 situation on aspects of diabetes management}

We developed 12 structured, 5-point Likert items to assess how, on a scale from 'much harder' to 'much easier', '... the Coronavirus (COVID-19) situation has impacted...' various socioeconomic, behavioural/clinical and psychosocial aspects of participants' diabetes management (past month). A neutral option (the pandemic has had no impact) was ordered in the middle between negative and positive response categories. Topics included drug affordability/accessibility, medication-taking behaviour, healthcare consultations, glucose monitoring and social support. Additionally, we incorporated two structured, binary items to assess drug rationing. See online supplemental appendix A for a complete list of these questions.

Sociodemographic and clinical characteristics of study sample

So as to align with the first administered COVID-19 subquestionnaire (analysed herein), sociodemographic and clinical characteristics were collated between the screening, baseline and follow-up questionnaires at months 1 and 2. Past-month frequencies of self-reported severe hypoglycaemia $(\mathrm{SH})$ and non-severe hypoglycaemia (NSH), defined in accordance with the ADA, ${ }^{22}$ were assessed at follow-up month 2. NSH was defined as any event that could be self-treated; $\mathrm{SH}$ was defined as a medical emergency that could not be self-treated (eg, required third-party assistance).

\section{Statistical analysis}

Categorical variables were summarised as frequencies and percentages, while continuous variables as means and SDs or medians and IQRs. Crude hypoglycaemia frequencies were calculated as incidence rates and proportions. Confirmed, probable and possible COVID-19 cases were calculated as 1-month period prevalences.

The impact of the COVID-19 situation on glycaemic management was descriptively analysed. Glycaemic management was operationalised according to different important aspects from drug affordability/accessibility to social support. Variability by diabetes type was assessed using the Wilcoxon-Mann-Whitney test for Likert responses and the two-sample test of proportions for binary responses. Tests were two sided at $\alpha=0.05$. All estimates were based on complete case analyses and were computed using STATA V.16.0.

\section{Patient and public involvement}

Neither the patients nor the public were directly involved in designing or conducting this study.

\section{RESULTS}

A total of 704 iNPHORM participants completed the first COVID-19 subquestionnaire (April 2020). Of these respondents, 667 (type 1 diabetes: 18.0\%; type 2 diabetes: $82 \%$ ) reported taking insulin and/or secretagogues (ie, met our study's eligibility criteria); their sociodemographic and clinical characteristics are summarised in tables 1 and 2, respectively.

Of the 667 eligible respondents, half were female. The mean age was 51.9 (SD: 14.6; min, max: 20, 87) years with $23.2 \% \geq 65$ years old. Diabetes duration was 26.0 (IQR: 23.0) years in people with type 1 diabetes and 11.0 (IQR: $14.0)$ years in people with type 2 diabetes. All respondents with type 1 diabetes and $38.4 \%$ with type 2 diabetes reported taking insulin without secretagogues; among the remaining participants with type 2 diabetes, 36.9\% were taking secretagogues without insulin and 24.7\% were taking a combination of insulin and secretagogues. Twenty-three per cent (type 1 diabetes: 23.3\%; type 2 
Table 1 Sociodemographic characteristics of study sample, overall and by diabetes type

\begin{tabular}{|c|c|c|c|}
\hline Sociodemographic characteristics & $\begin{array}{l}\text { Total } \\
\mathrm{n}=667\end{array}$ & $\begin{array}{l}\text { T1DM } \\
120 \text { (17.99\%) }\end{array}$ & $\begin{array}{l}\text { T2DM } \\
547(82.01 \%)\end{array}$ \\
\hline \multicolumn{4}{|l|}{ Age, mean (SD) } \\
\hline Years & $51.85(14.57)$ & $45.96(14.43)$ & $53.14(14.29)$ \\
\hline \multicolumn{4}{|l|}{ Age (categorical), n (\%) } \\
\hline$\geq 18$ and $\leq 40$ years & $180(26.99)$ & $51(42.50)$ & $129(23.58)$ \\
\hline$\geq 41$ and $\leq 64$ years & $332(49.78)$ & $53(44.17)$ & $279(51.01)$ \\
\hline$\geq 65$ and $\leq 74$ years & $123(18.44)$ & $11(9.17)$ & $112(20.48)$ \\
\hline$\geq 75$ years & $32(4.80)$ & $5(4.17)$ & $27(4.94)$ \\
\hline \multicolumn{4}{|l|}{ Sex assigned at birth, $\mathrm{n}(\%)$} \\
\hline Male & $326(48.88)$ & 40 (33.33) & $286(52.29)$ \\
\hline Female & $341(51.12)$ & $80(66.67)$ & $261(47.71)$ \\
\hline \multicolumn{4}{|l|}{ Race, n (\%) } \\
\hline White & 555 (83.21) & $111(92.50)$ & 444 (81.17) \\
\hline Black or African American & $52(7.80)$ & $3(2.50)$ & $49(8.96)$ \\
\hline Asian & $17(2.55)$ & $3(2.50)$ & $14(2.56)$ \\
\hline Hispanic, Latino/Latina or Spanish origin & $13(1.95)$ & $1(0.83)$ & $12(2.19)$ \\
\hline $\begin{array}{l}\text { American Indian, Alaska Native, Native } \\
\text { Hawaiian or Pacific Islander }\end{array}$ & $4(0.60)$ & 0 & $4(0.73)$ \\
\hline Multiracial & $23(3.45)$ & $2(1.67)$ & $21(3.84)$ \\
\hline Other & $3(0.45)$ & 0 & $3(0.55)$ \\
\hline \multicolumn{4}{|l|}{ Hispanic, Latino/Latina or Spanish origin, $\mathrm{n}(\%)$} \\
\hline Mexican, Mexican American, Chicano & $27(4.05)$ & $2(1.67)$ & $25(4.57)$ \\
\hline Puerto Rican & $6(0.90)$ & $1(0.83)$ & $5(0.91)$ \\
\hline Cuban & $2(0.30)$ & 0 & $2(0.37)$ \\
\hline $\begin{array}{l}\text { Other Hispanic, Latino/Latina or Spanish } \\
\text { origin }\end{array}$ & $3(0.45)$ & $1(0.83)$ & $2(0.37)$ \\
\hline $\begin{array}{l}\text { Not of Hispanic, Latino/Latina or } \\
\text { Spanish origin }\end{array}$ & $629(94.30)$ & $116(96.67)$ & $513(93.78)$ \\
\hline \multicolumn{4}{|l|}{ Highest level of education, $\mathrm{n}(\%)$} \\
\hline Elementary or high school (no diploma) & $10(1.50)$ & $3(2.50)$ & $7(1.28)$ \\
\hline $\begin{array}{l}\text { High school diploma or General } \\
\text { Educational Development (GED)/ } \\
\text { alternative credential }\end{array}$ & $101(15.14)$ & $19(15.83)$ & $82(14.99)$ \\
\hline College degree or some college & $425(63.72)$ & $75(62.50)$ & $350(63.99)$ \\
\hline $\begin{array}{l}\text { Degree beyond completing first college } \\
\text { bachelor's degree }\end{array}$ & $131(19.64)$ & $23(19.17)$ & $108(19.74)$ \\
\hline \multicolumn{4}{|l|}{ Current employment status, $\mathrm{n}(\%)$} \\
\hline $\begin{array}{l}\text { Employed full time or part-time } \\
\text { (including self-employment) }\end{array}$ & $346(51.87)$ & $73(60.83)$ & $273(49.91)$ \\
\hline $\begin{array}{l}\text { Temporarily laid off/temporarily } \\
\text { unemployed due to a health issue }\end{array}$ & $4(0.60)$ & $1(0.83)$ & $3(0.55)$ \\
\hline Unable to work due to disability & 84 (12.59) & $11(9.17)$ & 73 (13.35) \\
\hline Unemployed & $55(8.25)$ & $15(12.50)$ & $40(7.31)$ \\
\hline Student & $4(0.60)$ & $1(0.83)$ & $3(0.55)$ \\
\hline Retired & $174(26.09)$ & $19(15.83)$ & $155(28.34)$ \\
\hline \multicolumn{4}{|c|}{ Total annual household income (before taxes and deductions), $\mathrm{n}(\%)$} \\
\hline$<\$ 24999$ & 107 (16.21) & $13(11.21)$ & 94 (17.28) \\
\hline
\end{tabular}




\begin{tabular}{|c|c|c|c|}
\hline Sociodemographic characteristics & $\begin{array}{l}\text { Total } \\
\mathrm{n}=667\end{array}$ & $\begin{array}{l}\text { T1DM } \\
120(17.99 \%)\end{array}$ & $\begin{array}{l}\text { T2DM } \\
547(82.01 \%)\end{array}$ \\
\hline$\$ 25000$ to $\$ 54999$ & $173(26.21)$ & $25(21.55)$ & $148(27.21)$ \\
\hline$\$ 55000$ to $\$ 84999$ & $142(21.52)$ & 39 (33.62) & $103(18.93)$ \\
\hline$\$ 85000$ to $\$ 114999$ & 109 (16.52) & $22(18.97)$ & 87 (15.99) \\
\hline$\geq \$ 115000$ & $129(19.55)$ & $17(14.66)$ & $112(20.59)$ \\
\hline \multicolumn{4}{|l|}{ Current healthcare insurance, $\mathrm{n}(\%)$} \\
\hline $\begin{array}{l}\text { Insurance through a current or former } \\
\text { employer or union that is not a high- } \\
\text { deductible plan* }\end{array}$ & $153(22.94)$ & $36(30.00)$ & $117(21.39)$ \\
\hline $\begin{array}{l}\text { Insurance purchased directly from an } \\
\text { insurance company that is not a high- } \\
\text { deductible plan* }\end{array}$ & $49(7.35)$ & $11(9.17)$ & 38 (6.95) \\
\hline High-deductible plan* & $34(5.10)$ & $11(9.17)$ & $23(4.20)$ \\
\hline Medicare & $77(11.54)$ & $7(5.83)$ & $70(12.80)$ \\
\hline $\begin{array}{l}\text { Medicaid, Medical Assistance or other } \\
\text { government assistance plan }\end{array}$ & $74(11.09)$ & $17(14.17)$ & $57(10.42)$ \\
\hline TRICARE and Veterans Affairs & $9(1.35)$ & $2(1.67)$ & $7(1.28)$ \\
\hline Other & $5(0.75)$ & $2(1.67)$ & $3(0.55)$ \\
\hline Two or more insurance plans & $257(38.53)$ & $32(26.67)$ & $225(41.13)$ \\
\hline No insurance coverage at all & $9(1.35)$ & $2(1.67)$ & 7 (1.28) \\
\hline
\end{tabular}

Responses may not sum to total $(n=667)$ due to missing data.

*High-deductible plan: deductible $>\$ 1350$ for an individual or $>\$ 2700$ for a family.

T1DM, type 1 diabetes mellitus; T2DM, type 2 diabetes mellitus.

diabetes: $23.0 \%$ ) of the total sample reported A1c values $\geq 8.1 \%$. Sixty-one per cent reported $\geq 1$ diabetes-related complication(s) while $83.2 \%$ reported $\geq 1$ comorbidity.

Table 3 summarises self-reported hypoglycaemia incidences (combined daytime and nocturnal). The incidence rate and incidence proportion of NSH were higher in people with type 1 diabetes (incidence rate: 5.7 (95\% CI 4.6 to 7.1 ) events per person-month (PPM) and incidence proportion: $83.3 \%$ (95\% CI $75.7 \%$ to $88.9 \%)$ ) versus type 2 diabetes (incidence rate: 2.1 (95\% CI 1.8 to 2.4) events PPM and incidence proportion: $55.0 \%$ (95\% CI $50.8 \%$ to $59.1 \%)$ ). However, $\mathrm{SH}$, occurring at an overall rate of 0.7 (95\% CI 0.5 to 0.96$)$ events PPM, was almost twice as common in people with type 2 versus type 1 diabetes ( 0.8 (95\% CI 0.5 to 1.1 ) vs 0.4 (95\% CI 0.2 to 0.9$)$ events PPM). Similarly, the monthly incidence proportion of $\mathrm{SH}$, affecting nearly $13 \%$ (95\% CI $10.6 \%$ to $15.7 \%$ ) of respondents, was higher in people with type 2 diabetes compared with type 1 diabetes $(13.2 \%$ (95\% CI $10.6 \%$ to $16.3 \%$ ) vs $11.7 \%$ (95\% CI $7.08 \%$ to $18.6 \%$ )).

The 1-month period prevalences of confirmed, probable and possible COVID-19 were $0.75 \%$ (type 1 diabetes mellitus (T1DM): $\mathrm{n}=0$; type 2 diabetes mellitus (T2DM): $\mathrm{n}=5(0.75 \%)), 0.75 \%$ (T1DM: $\mathrm{n}=0$; T2DM: $\mathrm{n}=5(0.75 \%)$ ) and $8.9 \%$ (T1DM: $\mathrm{n}=16$ (13.33\%); T2DM: $\mathrm{n}=43$ (7.86\%)), respectively.
The impact of the COVID-19 situation on aspects of glycaemic management

A summary of results is provided in tables 4 and 5 . Almost a quarter of respondents (type 1 diabetes: $30.0 \%$; type 2 diabetes: $23.0 \%, \mathrm{p}=0.08$ ) reported that the COVID-19 situation made affording rent and other living expenses either 'somewhat harder' (type 1 diabetes: 19.2\%; type 2 diabetes: $14.6 \%$ ) or 'much harder' (type 1 diabetes: 10.8\%; type 2 diabetes: $8.4 \%$ ). Similarly, $27.6 \%$ (type 1 diabetes: $16.7 \%$; type 2 diabetes: $28.5 \%, \mathrm{p}=0.29$ ) of participants expressed it was 'somewhat harder' (type 1 diabetes: $16.7 \%$; type 2 diabetes: $20.1 \%$ ) or 'much harder' (type 1 diabetes: 6.7\%; type 2 diabetes: $8.4 \%$ ) to ensure adequate food supply to avoid hypoglycaemia. Close to one in five experienced challenges paying for their diabetes medications (type 1 diabetes: $16.7 \%$; type 2 diabetes: $19.0 \%, \mathrm{p}=0.71$ ) or test strips/sensors (type 1 diabetes: $13.3 \%$; type 2 diabetes: $18.3 \%, \mathrm{p}=0.38$ ); of these individuals, approximately half reported that their ability to afford therapeutic supplies had been made 'much harder' by the pandemic. Access-related issues were also identified. Overall, $27.4 \%$ (type 1 diabetes: $30.8 \%$; type 2 diabetes: $26.7 \%, \mathrm{p}=0.24)$ found the pandemic made it 'somewhat harder' (overall: 18.7\%; type 1 diabetes: $20.0 \%$; type 2 diabetes: $18.5 \%$ ) or 'much harder' (overall: 8.7\%; type 1 diabetes: $10.8 \%$; type 2 diabetes: $8.2 \%$ ) to retrieve diabetes medications from the pharmacy. As well, 
Table 2 Clinical characteristics of study sample, overall and by diabetes type

\begin{tabular}{|c|c|c|c|}
\hline Clinical characteristics & $\begin{array}{l}\text { Total } \\
n=667\end{array}$ & $\begin{array}{l}\text { T1DM } \\
120(17.99 \%)\end{array}$ & $\begin{array}{l}\text { T2DM } \\
547 \text { (82.01\%) }\end{array}$ \\
\hline \multicolumn{4}{|l|}{ Duration of diabetes, median (IQR) } \\
\hline Years & $13(15)$ & $26(23)$ & $11(14)$ \\
\hline \multicolumn{4}{|l|}{ Most recent haemoglobin A1c, $\mathrm{n}(\%)$} \\
\hline$\leq 7 \%$ & $252(37.78)$ & $45(37.50)$ & $207(37.84)$ \\
\hline $7.1 \%-8 \%$ & $239(35.83)$ & $45(37.50)$ & $194(35.47)$ \\
\hline $8.1 \%-9 \%$ & $99(14.84)$ & $14(11.67)$ & $85(15.54)$ \\
\hline$\geq 9.1 \%$ & $55(8.25)$ & $14(11.67)$ & $41(7.50)$ \\
\hline Unsure & $12(1.80)$ & 0 & $12(2.19)$ \\
\hline \multicolumn{4}{|l|}{ BMI at time of study enrolment, median (IQR) } \\
\hline $\mathrm{BMI}\left(\mathrm{kg} / \mathrm{m}^{2}\right)$ & $30.38(11.87)$ & $26.43(6.18)$ & 32.19 (11.99) \\
\hline \multicolumn{4}{|l|}{ Current insulin and/or secretagogue use, $\mathrm{n}(\%)$} \\
\hline Insulin without secretagogues & $330(49.48)$ & $120(100.00)$ & $210(38.39)$ \\
\hline Secretagogues without insulin & $202(30.28)$ & 0 & $202(36.93)$ \\
\hline Insulin in combination with secretagogues & $135(20.24)$ & 0 & $135(24.68)$ \\
\hline \multicolumn{4}{|c|}{ Diagnosed diabetes-related complications since 1 year preceding study enrolment, $n(\%)^{*}$} \\
\hline No complications & $263(39.43)$ & $41(34.17)$ & $222(40.59)$ \\
\hline One or more complications & $404(60.57)$ & $79(65.83)$ & $325(59.41)$ \\
\hline Amputation & $91(13.64)$ & $12(10.00)$ & $79(14.44)$ \\
\hline Diabetes ketoacidosis & $110(16.49)$ & $42(35.00)$ & $68(12.43)$ \\
\hline Foot damage & $123(18.44)$ & $18(15.00)$ & $105(19.20)$ \\
\hline Gastroparesis & $95(14.24)$ & $20(16.67)$ & $75(13.71)$ \\
\hline Hyperosmolar hyperglycaemic non-ketotic coma & $60(9.00)$ & $5(4.17)$ & 55 (10.05) \\
\hline Nephropathy & $114(17.09)$ & $18(15.00)$ & $96(17.55)$ \\
\hline Neuropathy & $298(44.68)$ & $46(38.33)$ & $252(46.07)$ \\
\hline Retinopathy & $156(23.39)$ & $46(38.33)$ & $110(20.11)$ \\
\hline \multicolumn{4}{|l|}{ Comorbidity status at time of study enrolment, $\mathrm{n}(\%)^{\star}$} \\
\hline No comorbidities & $112(16.79)$ & $32(26.67)$ & $80(14.63)$ \\
\hline One or more comorbidities & $555(83.21)$ & $88(73.33)$ & $467(85.37)$ \\
\hline Bone, joint or muscle problem & $310(46.48)$ & $39(32.50)$ & $271(49.54)$ \\
\hline Cancer & $52(7.80)$ & $3(2.50)$ & $49(8.96)$ \\
\hline Cardiovascular condition & $128(19.19)$ & $17(14.17)$ & $111(20.29)$ \\
\hline Chronic kidney disease & $73(10.94)$ & $8(6.67)$ & $65(11.88)$ \\
\hline Chronic liver failure or liver disease & $39(5.85)$ & $2(1.67)$ & $37(6.76)$ \\
\hline Eating disorder & $35(5.25)$ & $7(5.83)$ & $28(5.12)$ \\
\hline Gastrointestinal disease & $86(12.89)$ & $17(14.17)$ & 69 (12.61) \\
\hline HIV/AIDS & $11(1.65)$ & $2(1.67)$ & $9(1.65)$ \\
\hline Hypertension & $363(54.42)$ & $50(41.67)$ & $313(57.22)$ \\
\hline Mental health condition & $223(33.43)$ & $36(30.00)$ & $187(34.19)$ \\
\hline Neurological disorder & $39(5.85)$ & $8(6.67)$ & $31(5.67)$ \\
\hline Physical impairment & $168(25.19)$ & $29(24.17)$ & $139(25.41)$ \\
\hline Respiratory condition & $125(18.74)$ & $24(20.00)$ & $101(18.46)$ \\
\hline Stroke or transient ischaemic attack & $44(6.60)$ & $5(4.17)$ & $39(7.13)$ \\
\hline \multicolumn{4}{|l|}{ Current continuous glucose monitoring device use, $n(\%)$} \\
\hline Yes & $229(34.33)$ & $65(54.17)$ & $164(29.98)$ \\
\hline
\end{tabular}

Responses may not sum to total $(n=667)$ due to missing data.

${ }^{*}$ Cumulative percentage $>100 \%$ as participants could select more than one response.

BMI, body mass index; T1DM, type 1 diabetes mellitus; T2DM, type 2 diabetes mellitus. 
Table 3 Incidence rates and proportions of severe and non-severe hypoglycaemia, overall and by diabetes type

\begin{tabular}{|c|c|c|c|}
\hline Incidence rates* & $\begin{array}{l}\text { Total } \\
n=667\end{array}$ & $\begin{array}{l}\text { T1DM } \\
120(17.99 \%)\end{array}$ & $\begin{array}{l}\text { T2DM } \\
547(82.01 \%)\end{array}$ \\
\hline \multicolumn{4}{|l|}{ Severe hypoglycaemia (1 month retrospective) } \\
\hline Events per person-month $(95 \% \mathrm{Cl} \dagger)$ & $0.68(0.48$ to 0.96$)$ & $0.39(0.18$ to 0.85$)$ & 0.75 (0.51 to 1.09$)$ \\
\hline \multicolumn{4}{|c|}{ Non-severe hypoglycaemia (1 month retrospective) } \\
\hline \multicolumn{4}{|l|}{ Incidence proportions* } \\
\hline \multicolumn{4}{|l|}{ Severe hypoglycaemia (1 month retrospective) } \\
\hline \multicolumn{4}{|l|}{ Daytime or nocturnal } \\
\hline$\%$ with $\geq 1$ event $(95 \% \mathrm{Cl}$ ) & $60.06(56.29$ to 63.71$)$ & 83.33 (75.66 to 88.94$)$ & 54.95 (50.75 to 59.07$)$ \\
\hline
\end{tabular}

*Incidence rates and proportions are based on data from participants who reported taking insulin and/or secretagogues at month 2 follow-up. †Based on negative binomial distribution.

$\ddagger$ Based on Wilson score interval.

T1DM, type 1 diabetes mellitus; T2DM, type 2 diabetes mellitus.

because of the COVID-19 situation, $17 \%$ of participants reported rationing their diabetes medications either to make supplies last longer (type 1 diabetes: 13.3\%; type 2 diabetes: $17.4 \%, \mathrm{p}=0.28$ ) or avoid hypoglycaemia (overall: $16.8 \%$; type 1 diabetes: $15.8 \%$; type 2 diabetes: $17.0 \%$, $\mathrm{p}=0.76$ ).

The COVID-19 situation also influenced participants' abilities to self-manage. Many respondents struggled to remember to take their diabetes medication(s) as prescribed (overall: 13.7\%; type 1 diabetes: $6.7 \%$; type 2 diabetes: $15.2 \%, \mathrm{p}=0.047$ ) as well as test and monitor their blood glucose (overall: 15.9\%; type 1 diabetes: $5.0 \%$; type 2 diabetes: $18.3 \%, \mathrm{p}<0.001)$ and risk of hypoglycaemia regularly (overall: $12.0 \%$; type 1 diabetes: $7.5 \%$; type 2 diabetes: $13.0 \%, \mathrm{p}=0.026$ ). Over a third of respondents (type 1 diabetes: $35.0 \%$; type 2 diabetes: $36.8 \%, \mathrm{p}=0.75$ ) found it 'somewhat harder' (overall: 23.7\%; type 1 diabetes: $23.3 \%$; type 2 diabetes: $23.8 \%$ ) or 'much harder' (overall: 12.7\%; type 1 diabetes: $11.7 \%$; type 2 diabetes: $13.0 \%$ ) to consult with their diabetes care providers. In terms of exercise maintenance, almost one in two respondents (type 1 diabetes: $47.5 \%$; type 2 diabetes: $46.1 \%, \mathrm{p}=0.84$ ) reported that it had been 'somewhat harder' (overall: $31.3 \%$; type 1 diabetes: $30.0 \%$; type 2 diabetes: $31.6 \%$ ) or 'much harder' (overall: $15.0 \%$; type 1 diabetes: $17.5 \%$; type 2 diabetes: $14.4 \%$ ) to stay as physically active as usual.

Lastly, psychosocial effects were observed. Many participants (overall: $14.6 \%$; type 1 diabetes: $11.7 \%$; type 2 diabetes: $15.2 \%, \mathrm{p}=0.5$ ) felt the pandemic situation had made it 'somewhat harder' (overall: $9.3 \%$; type 1 diabetes: $7.5 \%$; type 2 diabetes: $9.7 \%$ ) or 'much harder' (overall:
5.3\%; type 1 diabetes: $4.2 \%$; type 2 diabetes: $5.5 \%$ ) to remain in control of their hypoglycaemia. Nineteen per cent also reported having insufficient social support to help manage their risk (type 1 diabetes: $10.8 \%$; type 2 diabetes: $20.3 \%, \mathrm{p}=0.056$ ); for $12.4 \%$ (type 1 diabetes: 8.3\%; type 2 diabetes: $13.4 \%$ ) accessing social support was 'somewhat harder', while for $6.2 \%$ (type 1 diabetes: $2.5 \%$; type 2 diabetes: $7.0 \%$ ) it was 'much harder'.

Although approximately $50 \%$ of respondents believed the pandemic situation had no impact on their glycaemic management, rarely was a beneficial impact on participants' lives observed. In general, less than $5 \%$ of the sample reported that the pandemic made aspects of their diabetes management either 'somewhat easier' or 'much easier'.

\section{DISCUSSION}

Experts have long been aware of the impacts a protracted emergency would have on healthcare and outcomes. Now, as two life-altering diseases collide, many Americans are finding themselves at the nidus of extreme clinical vulnerability, and with little support. Despite advice furnished by several national and international organisations, PWD are clearly struggling to maintain glycaemic management standards during the pandemic. This gap forebodes important, population-based consequences to diabetes-related morbidities, both now and well after vaccinations are distributed.

iNPHORM is the first investigation to quantify the impact of the COVID-19 situation on the socioeconomic, 
Table 4 Impact of the COVID-19 situation on aspects of participants' glycaemic management (past month)*, overall and by diabetes type

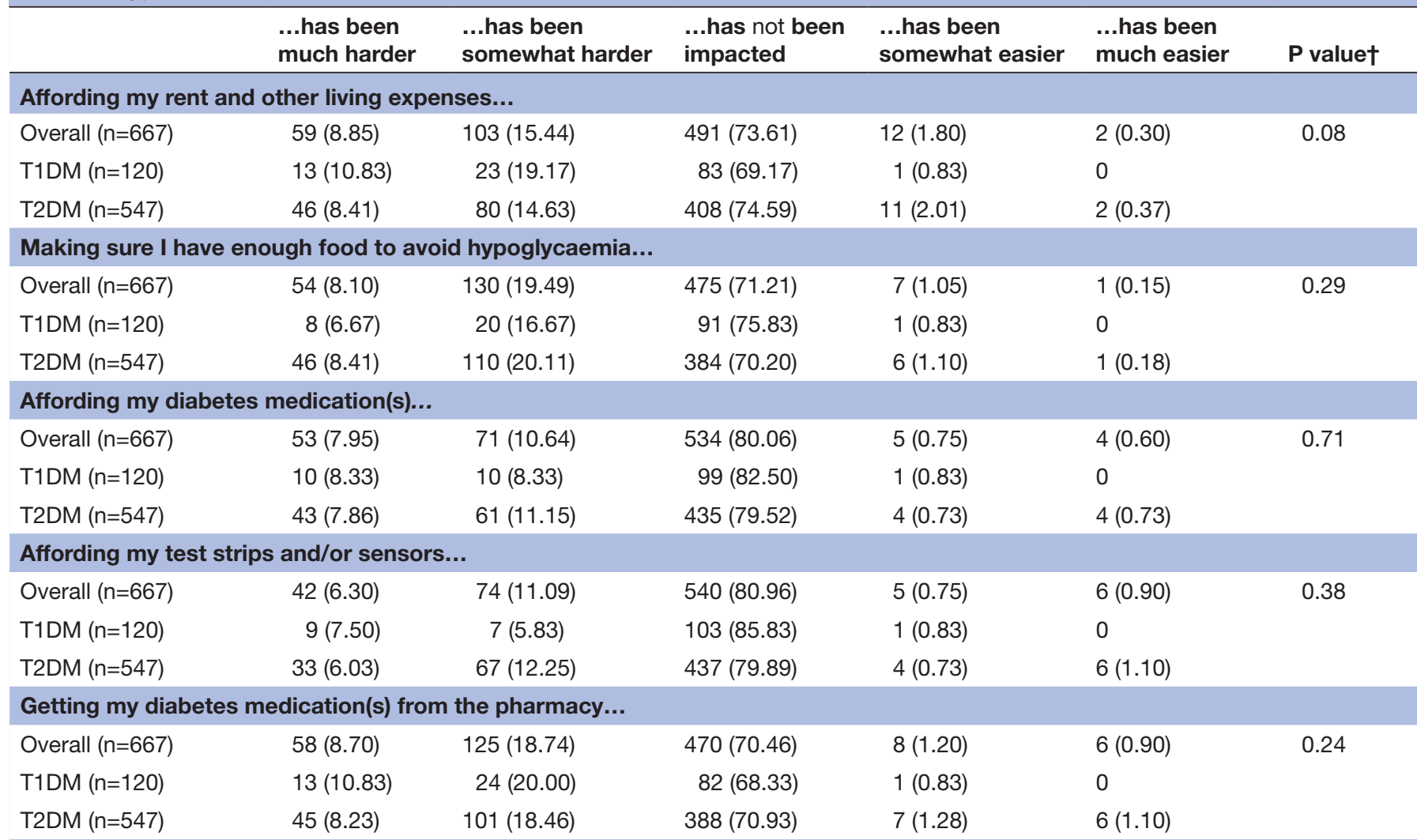

\section{Consulting with my healthcare provider(s) about my diabetes...}

\begin{tabular}{|c|c|c|c|c|c|c|}
\hline Overall $(n=667)$ & 85 (12.74) & 158 (23.69) & $410(61.47)$ & $13(1.95)$ & $1(0.15)$ & 0.75 \\
\hline T1DM $(n=120)$ & $14(11.67)$ & 28 (23.33) & 76 (63.33) & $2(1.67)$ & 0 & \\
\hline T2DM $(n=547)$ & $71(12.98)$ & $130(23.77)$ & 334 (61.06) & $11(2.01)$ & $1(0.18)$ & \\
\hline Overall $(n=667)$ & $34(5.10)$ & 72 (10.79) & $551(82.61)$ & 7 (1.05) & $3(0.45)$ & \multirow[t]{2}{*}{$<0.001 \ddagger$} \\
\hline T1DM $(n=120)$ & 4 (3.33) & $2(1.67)$ & $110(91.67)$ & $4(3.33)$ & 0 & \\
\hline
\end{tabular}

Remembering to take my diabetes medication(s) as prescribed...

\begin{tabular}{|c|c|c|c|c|c|c|}
\hline Overall (n=667) & $26(3.90)$ & $65(9.75)$ & 554 (83.06) & $18(2.70)$ & $4(0.60)$ & $0.047 \ddagger$ \\
\hline T1DM (n=120) & $1(0.83)$ & $7(5.83)$ & $109(90.83)$ & $3(2.50)$ & 0 & \\
\hline T2DM $(n=547)$ & $25(4.57)$ & $58(10.60)$ & $445(81.35)$ & $15(2.74)$ & $4(0.73)$ & \\
\hline Overall (n=667) & $29(4.35)$ & $51(7.65)$ & $561(84.11)$ & $23(3.45)$ & $3(0.45)$ & \multirow[t]{2}{*}{$0.026 \ddagger$} \\
\hline T1DM (n=120) & $3(2.50)$ & $6(5.00)$ & 103 (85.83) & $8(6.67)$ & 0 & \\
\hline Overall (n=667) & 100 (14.99) & 209 (31.33) & 329 (49.33) & $23(3.45)$ & $6(0.90)$ & \multirow[t]{3}{*}{0.84} \\
\hline T1DM $(n=120)$ & $21(17.50)$ & $36(30.00)$ & $55(45.83)$ & $7(5.83)$ & $1(0.83)$ & \\
\hline T2DM $(n=547)$ & $79(14.44)$ & $173(31.63)$ & $274(50.09)$ & $16(2.93)$ & $5(0.91)$ & \\
\hline \multicolumn{7}{|c|}{ Feeling in control of my hypoglycaemia... } \\
\hline
\end{tabular}

Having enough social support to help me manage my hypoglycaemia... 
Table 4 Continued

\begin{tabular}{lcllll} 
& $\begin{array}{c}\text {...has been } \\
\text { much harder }\end{array}$ & $\begin{array}{l}\text {...has been } \\
\text { somewhat harder }\end{array}$ & $\begin{array}{l}\text {...has not been } \\
\text { impacted }\end{array}$ & $\begin{array}{l}\text {...has been } \\
\text { somewhat easier }\end{array}$ & $\begin{array}{l}\text {...has been } \\
\text { much easier }\end{array}$ \\
\hline Overall $(n=667)$ & $41(6.15)$ & $83(12.44)$ & $518(77.66)$ & $21(3.15)$ & $4(0.60)$ \\
T1DM valuet $(n=120)$ & $3(2.50)$ & $10(8.33)$ & $104(86.67)$ & $3(2.50)$ & 0 \\
T2DM $(n=547)$ & $38(6.95)$ & $73(13.35)$ & $414(75.69)$ & $18(3.29)$ & $4(0.73)$
\end{tabular}

$\mathrm{n}(\%)$ are presented.

*Data collected from 21 to 28 April 2020.

†ltem responses were compared between individuals with T1DM and T2DM. P values were computed using two-sample Wilcoxon-MannWhitney tests.

$\ddagger$ Statistically significant at $\alpha=0.05$ (ie, the underlying distributions of item responses statistically significantly differed by diabetes type).

T1DM, type 1 diabetes mellitus; T2DM, type 2 diabetes mellitus.

behavioural/clinical and psychosocial aspects of glycaemic management among community-dwelling Americans. Based on the results of our study, the pandemic was found to cause substantial deficiencies in routine diabetes care in the USA, a finding consistent with international data published by the WHO. ${ }^{23}$ Of note, only few appreciable differences were observed by diabetes type; of those identified, most related to the behavioural aspects of glycaemic management.

\section{COVID-19 and the socioeconomic aspects of glycaemic management}

PWD have been severely and disproportionately affected by the pandemic. Based on recent data published by the ADA, 24\% of PWD have been forced to use savings, loans or money from their stimulus checks. ${ }^{24}$ This percentage increases to half among the $33 \%$ of PWD (compared with $29 \%$ of people without diabetes) who have lost income since the pandemic began. ${ }^{24}$ It is thus not surprising that almost a quarter of iNPHORM respondents revealed that the COVID-19 situation impeded their abilities to afford rent and other living expenses. As the outbreak continues to escalate across the country, it is expected that the

Table 5 Impact of the COVID-19 situation on diabetes medication rationing (past month) ${ }^{*}$, overall and by diabetes type

$\begin{array}{llll} & \text { T1DM } & \text { T2DM } & \\ \text { Total } & 120 & 547 & \\ \mathrm{n}=667 & (17.99 \%) & (82.01 \%) & \text { P value }\end{array}$

\begin{tabular}{|c|c|c|c|c|}
\hline \multicolumn{5}{|c|}{$\begin{array}{l}\text { Rationed to make diabetes medication(s) } \\
\text { supply last longer }\end{array}$} \\
\hline Yes & $111(16.64)$ & $16(13.33)$ & $95(17.37)$ & 0.28 \\
\hline \multicolumn{5}{|c|}{ Rationed to avoid hypoglycaemia } \\
\hline Yes & 112 (16.79) & 19 (15.83) & $93(17.00)$ & 0.76 \\
\hline
\end{tabular}

$\mathrm{n}(\%)$ are presented.

*Data collected from 21 to 28 April 2020.

†Item responses were compared between individuals with T1DM and T2DM. $P$ values were computed using two-sample $Z$ tests for proportions.

T1DM, type 1 diabetes mellitus; T2DM, type 2 diabetes mellitus. financial situation of many Americans will become increasingly precarious. ${ }^{18}$

In this study, economic incertitude also affected participants' access to healthy food. ${ }^{9}$ COVID-19-related financial or environmental factors can invoke a state of food insecurity, a major predictor of clinically significant hypoglycaemia. ${ }^{25}$ One US study found that exhaustion of food budgets was associated with a $27 \%$ increase in hypoglycaemia-related hospital admissions. ${ }^{26}$ Food insecurity among PWD has also been associated with poorer glucose monitoring and higher A1c values. ${ }^{26}$

Furthermore, decreases in financial resources, especially in the absence of health coverage, can inhibit access to diabetes medical supplies. An American study found that prescription refills for diabetes medications fell by $10 \%$ between January and August $2020^{27}$; however, whether or not this was due to financial or environmental factors was unclarified. Our data reveal that while roughly $20 \%$ of respondents experienced difficulties affording medications or strips/sensors, over a quarter reported issues physically retrieving medical supplies from pharmacies (perhaps due to prevention orders or anxieties over potential exposure).

Interruptions in healthcare access may explain the significant percentages of respondents who reported rationing their diabetes supplies. Our study investigated whether or not PWD ration their medications not just to extend their lifespan, but to prevent hypoglycaemia. Despite evidence that lockdown exacerbates hypoglycaemia risk, ${ }^{28}$ no research yet existed measuring the potential risk of hypoglycaemia-specific medication rationing during COVID-19. Treatment rationing contradicts the CDC's recommendations for managing diabetes during the pandemic. ${ }^{11}$ Antihyperglycaemic underuse can increase the likelihood of deleterious short-term outcomes, and it can drive up the cost of long-term diabetes-related complications. ${ }^{29}$

The impact of the COVID-19 situation on socioeconomic indicators predictably did not vary by diabetes type with nearly equivalent percentages of each reporting financial and environmental instabilities because of the pandemic. 
COVID-19 and the behavioural/clinical aspects of glycaemic management

Evidence from past national emergencies underscores their profound and lasting implications on selfmanagement behaviours in people with coexistent illnesses. ${ }^{1617}$ Our study is the first American diabetes investigation to measure these implications in the COVID-19 era. Because of the pandemic, several iNPHORM participants reported forgetting to take their prescribed medications. This was especially true of type 2 diabetes respondents, perhaps due to variability in medication regimens compared with those with type 1 diabetes. Lapses in medication use can compromise therapeutic adherence and efficacies, leading to elevated A1c values as far-out as 16 months postemergency. ${ }^{17}$ This risk is likely compounded by financial and access-related issues resulting from the pandemic (described in previous section) as well as suboptimal blood glucose tracking. Many respondents, especially those with type 2 diabetes, reported difficulties testing/monitoring their glucose and, specifically, hypoglycaemia risk.

In addition, the pandemic has imposed dramatic changes on routine healthcare access and delivery, particularly among individuals with underlying health conditions. ${ }^{30}$ To prioritise access to hospital beds, equipment and staff, as well as to minimise viral transmission, much of routine healthcare has been postponed or cancelled. As well, PWD may decline attendance at hospitals, clinics and screening examinations over concerns of infection. More than a third of respondents indicated that the COVID-19 situation made it harder to consult with their diabetes providers. Interestingly, this finding did not significantly differ by diabetes type.

Research has shown that deferred or avoided healthcare due to the pandemic can contribute to excess morbidity and mortality. ${ }^{31}$ Based on an article by Woolf et $a l,{ }^{32} \mathrm{US}$ states with large numbers of COVID-19-related deaths experience large proportional increases in deaths from other underlying causes, including diabetes. Impacts on health may worsen the longer community containment measures last. A simulation study of data from previous global disasters found the duration of lockdown to be directly proportional to A1c and number of diabetesrelated complications. ${ }^{33}$ Unfortunately, these effects may endure even after the viral outbreak has been quelled. Evidence from past disasters has shown that reduced access to healthcare during the acute phase of an emergency can lead to an aftermath of increased deaths and morbidities including stroke, myocardial infarctions and diabetesrelated complications. ${ }^{34}$ Such increases in morbidity and mortality resulting from delayed and reduced healthcare access are especially concerning among iNPHORM participants, of whom almost $90 \%$ reported some comorbidity or diabetes-related complication.

Finally, COVID-19 mitigation measures can restrict access to indoor and outdoor physical activities, contributing to increased sedentary behaviours that adversely affect immune defence, glycaemic control and metabolic health in general. ${ }^{9}$ Based on data from other viral infections, suboptimal physical activity can accentuate symptom severity, recovery times and transmissibility; it can also compromise postvaccination immunity and increase secondary infection risk. ${ }^{35}$ Regardless of diabetes type, staggering percentages of participants reported reduced physical activity because of the pandemic, a sure warning sign of the extensive health consequences to come.

\section{COVID-19 and the psychosocial aspects of glycaemic management}

The psychosocial ramifications of COVID-19 in PWD have been minimally investigated in the literature. Our study specifically assessed how the pandemic has impacted respondents' senses of personal control over their hypoglycaemia risk. Significant decrements in selfperceived control were observed across all participants. Sense of control-the learnt belief that one does master, control and shape one's life-has been linked to several positive health effects including proactive behaviour and emotional well-being. ${ }^{36}$ However, inadequate supplies, financial loss, fear psychosis of being infected and media/ disinformation can all contribute to increased feelings of powerlessness. ${ }^{37}$ Reductions in sense of personal control have been associated with heightened stress, anxiety and depressive symptoms ${ }^{38}$-outcomes that have been linked to poor medication adherence and diminished self-management. ${ }^{39}$

No study had yet quantified the effect of the COVID-19 situation on social support access in PWD. While support from family and friends can mediate the contextual impacts of COVID-19, several respondents in our study, particularly those with type 2 diabetes, reported insufficient social support to help manage their hypoglycaemia. Assistance from informal relationships has been identified as a major component to hypoglycaemia self-management with demonstrable impacts on diabetes-related morbidity and mortality reduction. ${ }^{14840}$ The gap in social support observed in our study portends troubling implications for hypoglycaemia incidence as well as other clinical and psychosocial sequelae.

Indeed, suboptimal social support among people with type 2 diabetes, compounded by inadequate hypoglycaemia risk monitoring, could explain why SH was found to be more common in our respondents with type 2 versus type 1 diabetes. Though comparable overall hypoglycaemia incidences have been observed in other realworld studies, ${ }^{41}$ the 2018 UnderstandINg the impact of HYPOglycemia on Diabetes Management (InHypo-DM) study ${ }^{42}$ reported similarly higher $\mathrm{SH}$ event rates in people with type 2 versus type 1 diabetes. This finding suggests that important deficiencies-irrespective of the pandemic situation-may exist with regard to hypoglycaemia education, management behaviours and/or primary care in people with type 2 diabetes when compared with their type 1 diabetes counterpart. Parenthetically, unlike many other real-world hypoglycaemia investigations that focus exclusively on insulin-treated diabetes, ${ }^{43-45}$ it should be 
noted that $25 \%$ and $18 \%$ of participants in iNPHORM and InHypo-DM, respectively, reported taking insulin in combination with secretagogues. Research has shown that insulin-secretagogue therapy substantially increases the rate of SH compared with insulin without secretagogues and secretagogues without insulin. ${ }^{46}$

\section{Study strengths and limitations}

This study evaluates a general, community-based cohort of Americans with diabetes-irrespective of infection status-to derive insight into the real-world, real-time consequences of the COVID-19 situation in diabetes. To mitigate selection bias, a broad sample of participants was recruited from a large, probability-based internet panel. Online data collection enabled us to capitalise on the high prevalence of internet use in the USA, ${ }^{47}$ while optimising survey reach and accessibility, respondent honesty and representativeness, as well as reducing item non-response. ${ }^{48} 49$ Participant anonymity and confidentiality were assured to decrease the risk of social desirability bias. $^{50}$

By developing a pandemic-specific questionnaire, our research team was able to elucidate the once unknown repercussions of the COVID-19 situation in Americans with diabetes; indeed, self-report data can offer unique and robust insight routinely uncaptured by other methods. Though the study is cross-sectional in design, self-reported causal attributions of the pandemic were operationalised for each questionnaire item: respondents were asked to indicate to what extent '...the Coronavirus (COVID-19) situation [had] impacted' given aspects of glycaemic management. Such information had yet to be garnered in the USA.

Nevertheless, certain study limitations should be noted. Selection biases may have arisen to the extent that respondents differed non-randomly from the general US population with diabetes taking insulin and/or secretagogues. Specifically, survivorship and coverage bias (eg, due to high observed percentages of Caucasian, educated and insured participants) could have curtailed the external validity of results. Volunteer bias may have also led participants to overestimate or underestimate their responses. For example, those who chose to complete the first COVID-19 subquestionnaire may have possessed systemically different (positive or negative) pandemic-related perspectives and/or experiences than those who did not.

Estimates derived in our study may be conservative, as they stem from a 1-month data capture in the early phase of the pandemic trajectory. In addition, selfreported responses could have been influenced by social desirability bias and/or recall error. Even so, self-report data-typically the Hobson's choice for information on perspectives, views and opinions-enabled us to capture, for the first time, the impacts of COVID-19 on various socioeconomic, behavioural/clinical and psychosocial aspects of diabetes management in the USA. The results of our study (though not exhaustive, per se) provide important, unprecedented insight into the real-world fallouts of the pandemic situation on diabetes-related health.

Analyses of psychometric properties and/or adjusted frequency estimates were beyond the scope of this manuscript. Rather this study supplies descriptive, novel and time-sensitive evidence at the convergence of COVID-19 and diabetes, contributing to both the national and international body of pandemic literature.

\section{CONCLUSIONS}

A 'hinterland' is defined as an area lying beyond what is visible or known. As a society, we have exhibited unparalleled bravery in the face of one of the most terrifying crises known to humankind. However, our mission to abate the pandemic is only just beginning. Indeed, the COVID-19 calamity has had untold reverberations in the lives of Americans, extending well beyond the visible devastations caused by infection alone. Not least are the impacts COVID-19 has had on PWD—cases and non-cases alike-who have struggled to maintain control of their disease amidst the pandemic.

Yet, until now, the nature and scale of these impacts in the USA were largely unknown or uncharacterised. Thus, the results of our study draw awareness to the far-reaching and potential lasting consequences of the pandemic, and offer an evidence base for decisive action. In identifying the unique needs of Americans with diabetes during the COVID-19 era, we can begin to develop, implement and assess clinical and public health strategies that ensure safe, undisrupted care within communities of PWD. As we combat the acute phase of COVID-19, we must not lose sight of the pernicious health challenges that coexist and await us in the aftermath.

Acknowledgements We thank Nadia Khan and Kristina Timcevska for their valuable feedback on this manuscript.

Contributors AAR-L contributed to the discussion, researched the data and wrote the manuscript. BLR, JDB and JWD contributed to the discussion and reviewed/ edited the manuscript. JEB reviewed/edited the manuscript. SBH contributed to the discussion, researched the data and reviewed/edited the manuscript. SBH and AAR- $L$ are the guarantors of the study.

Funding Funding for this study was provided through an investigator-initiated grant from Sanofi Global.

Disclaimer Sanofi Global was not involved in the study design; in the collection, analysis and interpretation of data; in the writing of the report; and in the decision to submit the article for publication. All researchers confirm their independence from the funder.

Competing interests AAR-L: Sanofi: grant; Eli Lilly: consultant; fees paid for presentations; Novo Nordisk: consultant. SBH: Sanofi: grant, member advisory board, consultant; Eli Lilly: grant, member advisory board, consultant, clinical studies; Novo Nordisk: grant, member advisory board, consultant, clinical studies; Janssen: grant, member advisory board, consultant; AstraZeneca: grant, member advisory board, consultant, clinical studies; Abbott: grant, member advisory board, consultant; Boehringer Ingelheim: grant, member advisory board, consultant, clinical studies; JDRF: grant; Lawson: grant; Canadian Institutes of Health and Research: grants.

Patient consent for publication Not required.

Ethics approval Western investigators and IIS obtained ethics approval from the Western University's Research Ethics Board and the Pearl Institutional Review Board 
(USA), respectively (ID: 112986). Participants gave informed consent before taking part in the study.

Provenance and peer review Not commissioned; externally peer reviewed.

Data availability statement № data are available.

Supplemental material This content has been supplied by the author(s). It has not been vetted by BMJ Publishing Group Limited (BMJ) and may not have been peer-reviewed. Any opinions or recommendations discussed are solely those of the author(s) and are not endorsed by BMJ. BMJ disclaims all liability and responsibility arising from any reliance placed on the content. Where the content includes any translated material, BMJ does not warrant the accuracy and reliability of the translations (including but not limited to local regulations, clinical guidelines, terminology, drug names and drug dosages), and is not responsible for any error and/or omissions arising from translation and adaptation or otherwise.

Open access This is an open access article distributed in accordance with the Creative Commons Attribution Non Commercial (CC BY-NC 4.0) license, which permits others to distribute, remix, adapt, build upon this work non-commercially, and license their derivative works on different terms, provided the original work is properly cited, appropriate credit is given, any changes made indicated, and the use is non-commercial. See: http://creativecommons.org/licenses/by-nc/4.0/.

\section{ORCID iD}

Stewart B Harris http://orcid.org/0000-0002-1794-6551

\section{REFERENCES}

1 Holshue ML, DeBolt C, Lindquist S, et al. First case of 2019 novel coronavirus in the United States. N Engl J Med 2020;382:929-36.

2 Johns Hopkins. Coronavirus Resource Center [Internet]. Johns Hopkins Coronavirus Resource Center. Available: https://coronavirus. jhu.edu/ [Accessed 26 Dec 2020].

3 Roncon L, Zuin M, Rigatelli G, et al. Diabetic patients with COVID-19 infection are at higher risk of ICU admission and poor short-term outcome. J Clin Virol 2020;127:104354.

4 Hill MA, Mantzoros C, Sowers JR. Commentary: COVID-19 in patients with diabetes. Metabolism 2020;107:154217.

5 Gupta R, Ghosh A, Singh AK, et al. Clinical considerations for patients with diabetes in times of COVID-19 epidemic. Diabetes Metab Syndr 2020;14:211-2.

6 Brownlee M. Biochemistry and molecular cell biology of diabetic complications. Nature 2001;414:813-20.

7 Morra ME, Van Thanh L, Kamel MG, et al. Clinical outcomes of current medical approaches for middle East respiratory syndrome: a systematic review and meta-analysis. Rev Med Virol 2018;28:e1977.

8 Ma RCW, Holt RIG. COVID-19 and diabetes. Diabet Med 2020;37:723-5.

9 Singh AK, Gupta R, Ghosh A, et al. Diabetes in COVID-19: prevalence, pathophysiology, prognosis and practical considerations. Diabetes Metab Syndr 2020;14:303-10.

10 Geerlings SE, Hoepelman Al. Immune dysfunction in patients with diabetes mellitus (DM). FEMS Immunol Med Microbiol 1999;26:259-65.

11 CDC. COVID-19 and Your Health [Internet]. Centers for Disease Control and Prevention, 2020. Available: https://www.cdc.gov/ coronavirus/2019-ncov/need-extra-precautions/people-withmedical-conditions.html [Accessed 26 Dec 2020].

12 Diabetes and Coronavirus (COVID-19) | ADA [Internet]. Available: https://www.diabetes.org/coronavirus-covid-19 [Accessed 26 Dec 2020].

13 International Diabetes Federation. Coronavirus COVID-19, 2020. Available: https://www.idf.org/aboutdiabetes/what-is-diabetes/covid19-and-diabetes/1-covid-19-and-diabetes.html [Accessed 26 Dec 2020].

14 McEwen MM, Pasvogel A, Gallegos G, et al. Type 2 diabetes selfmanagement social support intervention at the U.S.-Mexico border. Public Health Nurs 2010;27:310-9.

15 Yang Y, Shang W, Rao X. Facing the COVID-19 outbreak: what should we know and what could we do? J Med Virol 2020;92:536-7.

16 Fonseca VA, Smith H, Kuhadiya N, et al. Impact of a natural disaster on diabetes: exacerbation of disparities and long-term consequences. Diabetes Care 2009;32:1632-8.

$17 \mathrm{Ng} \mathrm{J}$, Atkin SL, Rigby AS, et al. The effect of extensive flooding in Hull on the glycaemic control of patients with diabetes. Diabet Med 2011;28:519-24.

18 dQ\&A. Impact of COVID-19 on the diabetes community in the United States, 2020 [Internet]. Available: https://d-qa.com/impact-of-covid19-on-the-usa-diabetes-community/ [Accessed 24 Dec 2020].
19 Qiu J, Shen B, Zhao M, et al. A nationwide survey of psychological distress among Chinese people in the COVID-19 epidemic: implications and policy recommendations. Gen Psychiatr 2020;33:e100213.

20 Harris S. Investigating Novel Predictions of Hypoglycemia Occurrence in Real-world Models [Internet]. clinicaltrials.gov (Report No.: NCT04219514), 2020. Available: https://clinicaltrials.gov/ct2/ show/NCT04219514 [Accessed 23 Dec 2020].

21 Coronavirus disease 2019 (COVID-19) | 2020 interim case definition. Available: https://ndc.services.cdc.gov/case-definitions/coronavirusdisease-2019-2020-08-05/ [Accessed 24 Dec 2020].

22 American Diabetes Association. 6. Glycemic Targets: Standards of Medical Care in Diabetes-2019. Diabetes Care 2019;42:S61-70.

23 COVID-19 significantly impacts health services for noncommunicable diseases [Internet]. Available: https://www.who.int/news/item/ 01-06-2020-covid-19-significantly-impacts-health-services-fornoncommunicable-diseases [Accessed 05 Jul 2020].

24 dQ\&A - The Diabetes Research Company and the, American Diabetes Association. Diabetes and COVID 19:New Data Quantifies Extraordinary Challenges Faced by Americans with Diabetes During Pandemic, 2020. https://www.diabetes.org/sites/default/files/202007/7.29.2020 dQA-ADA\%20Data\%20Release.pdf

25 Seligman HK, Jacobs EA, Lopez A, et al. Food insecurity and hypoglycemia among safety net patients with diabetes. Arch Intern Med 2011;171:1204-6.

26 Seligman HK, Bolger AF, Guzman D, et al. Exhaustion of food budgets at month's end and hospital admissions for hypoglycemia. Health Aff 2014;33:116-23.

27 Hartmann-Boyce J, Morris E, Goyder C, et al. Diabetes and COVID-19: risks, management, and Learnings from other national disasters. Diabetes Care 2020;43:1695-703.

28 Shah K, Tiwaskar M, Chawla P, et al. Hypoglycemia at the time of Covid-19 pandemic. Diabetes Metab Syndr 2020;14:1143-6.

29 Cutler RL, Fernandez-Llimos F, Frommer M, et al. Economic impact of medication non-adherence by disease groups: a systematic review. BMJ Open 2018;8:e016982.

30 Propper C, Stockton I, Stoye G. COVID-19 and disruptions to the health and social care of older people in England [Internet], 2020. Available: https://www.ifs.org.uk/publications/15160 [Accessed 24 Dec 2020].

31 Center For Disease Control and Prevention. . Excess Deaths Associated with COVID-19 [Internet]., 2020. Available: https://www. cdc.gov/nchs/nvss/vsrr/covid19/excess_deaths.htm [Accessed 24 Dec 2020].

32 Woolf SH, Chapman DA, Sabo RT, et al. Excess deaths from COVID-19 and other causes, March-April 2020. JAMA 2020;324:510-3.

33 Ghosal S, Sinha B, Majumder M, et al. Estimation of effects of nationwide lockdown for containing coronavirus infection on worsening of glycosylated haemoglobin and increase in diabetesrelated complications: a simulation model using multivariate regression analysis. Diabetes Metab Syndr 2020;14:319-23.

34 Mokdad AH, Mensah GA, Posner SF, et al. When chronic conditions become acute: prevention and control of chronic diseases and adverse health outcomes during natural disasters. Prev Chronic Dis 2005;2 Spec no:A04

35 Pascoe AR, Fiatarone Singh MA, Edwards KM. The effects of exercise on vaccination responses: a review of chronic and acute exercise interventions in humans. Brain Behav Immun 2014;39:33-41.

36 Mirowsky J, Ross CE. Eliminating defense and agreement bias from measures of the sense of control: a $2 \times 2$ index. Soc Psychol Q 1991;54:127-45

37 Brooks SK, Webster RK, Smith LE, et al. The psychological impact of quarantine and how to reduce it: rapid review of the evidence. The Lancet 2020;395:912-20.

38 Keeton CP, Perry-Jenkins M, Sayer AG. Sense of control predicts depressive and anxious symptoms across the transition to parenthood. J Fam Psychol 2008;22:212-21.

39 Sturt J, Dennick K, Due-Christensen M, et al. The detection and management of diabetes distress in people with type 1 diabetes. Curr Diab Rep 2015;15:101.

40 Nicklett EJ, Liang J. Diabetes-Related support, regimen adherence, and health decline among older adults. J Gerontol B Psychol Sci Soc Sci 2010;65B:390-9.

41 Edridge CL, Dunkley AJ, Bodicoat DH, et al. Prevalence and incidence of hypoglycaemia in 532,542 people with type 2 diabetes on oral therapies and insulin: a systematic review and meta-analysis of population based studies. PLoS One 2015;10:e0126427.

42 Ratzki-Leewing A, Harris SB, Mequanint S, et al. Real-world crude incidence of hypoglycemia in adults with diabetes: results 
of the InHypo-DM study, Canada. BMJ Open Diabetes Res Care 2018;6:e000503.

43 Leiter L, Yale J, Chiasson J. Assessment of the impact of fear of hypoglycemic episodes on glycemic and hypoglycemia management. Can J Diabetes 2005;29:186-92.

44 Leiter LA, Boras D, Woo VC. Dosing irregularities and self-treated hypoglycemia in type 2 diabetes: results from the Canadian cohort of an international survey of patients and healthcare professionals. Can J Diabetes 2014;38:38-44.

45 Aronson R, Goldenberg R, Boras D, et al. The Canadian hypoglycemia assessment tool program: insights into rates and implications of hypoglycemia from an observational study. Can J Diabetes 2018;42:11-17.

46 Mogensen UM, Andersson C, Fosbøl EL, et al. Sulfonylurea in combination with insulin is associated with increased mortality compared with a combination of insulin and metformin in a retrospective Danish nationwide study. Diabetologia 2015;58:50-8.

47 Pew Research Center. Demographics of Internet and Home Broadband Usage in the United States [Internet]. Pew Research Center: Internet, Science \& Tech, 2021. Available: https://www. pewresearch.org/internet/fact-sheet/internet-broadband/ [Accessed 4 May 2021].

48 Warner CH, Appenzeller GN, Grieger T, et al. Importance of anonymity to encourage honest reporting in mental health screening after combat deployment. Arch Gen Psychiatry 2011;68:1065-71.

49 Gnambs T, Kaspar K. Disclosure of sensitive behaviors across selfadministered survey modes: a meta-analysis. Behav Res Methods 2015;47:1237-59.

50 Althubaiti A. Information bias in health research: definition, pitfalls, and adjustment methods. J Multidiscip Healthc 2016;9:211-7. 